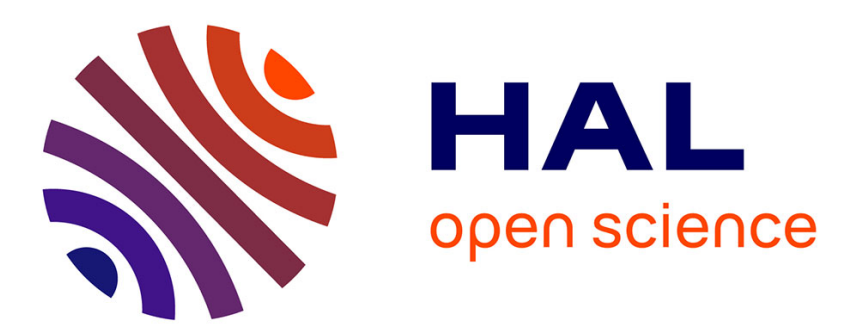

\title{
La fabrique urbaine de Tours: une analyse dialectique entre sociétés et espaces urbains (IXe-XIIIe siècle)
}

Hélène Noizet

\section{To cite this version:}

Hélène Noizet. La fabrique urbaine de Tours: une analyse dialectique entre sociétés et espaces urbains (IXe-XIIIe siècle). La fabrique urbaine de Tours: une analyse dialectique des relations entre sociétés et espaces urbains, Dec 2004, Bruxelles, Belgique. pp.19-37. halshs-00218276

\section{HAL Id: halshs-00218276 \\ https://shs.hal.science/halshs-00218276}

Submitted on 26 Jan 2008

HAL is a multi-disciplinary open access archive for the deposit and dissemination of scientific research documents, whether they are published or not. The documents may come from teaching and research institutions in France or abroad, or from public or private research centers.
L'archive ouverte pluridisciplinaire HAL, est destinée au dépôt et à la diffusion de documents scientifiques de niveau recherche, publiés ou non, émanant des établissements d'enseignement et de recherche français ou étrangers, des laboratoires publics ou privés. 
Noizet, Hélène, "La fabrique urbaine de Tours : une analyse dialectique entre sociétés et espaces urbains (IXe-XIII ${ }^{\mathrm{e}}$ siècle)", dans Voisinages, coexistences, appropriations. Groupes sociaux et territoires urbains (Moyen Âge - 16 siècle), édité par Chloé Deligne et Claire Billen, p. 19-37, Turnhout, Brepols, 2007.

\section{LA FABRIQUE URBAINE DE TOURS : UNE ANALYSE DIALECTIQUE ENTRE SOCIÉTÉS ET ESPACES URBAINS (IX ${ }^{\mathrm{E}}$-XIII ${ }^{\mathrm{E}}$ SIÈCLE)}

\section{Hélène Noizet}

Université de Paris-I

Auteur : Hélène Noizet

Mail : helene.noizet@univ-paris1.fr

Adresse personnelle : 33 rue Parmentier, 59370 Mons-en-Baroeul. Tel : 03205611 98

situation et adresse professionnelle : maitre de conférences en histoire médiévale à l'université de Paris-1 Panthéon-Sorbonne, 17 rue de la Sorbonne, 75005 Paris laboratoire de rattachement: UMR 8589 LAMOP, 1 rue Victor Cousin, 75005 Paris.

Résumé : À Tours, l'espace urbain ne semble pas résulter d'un projet planifié. Si l'analyse morphologique permet de repérer des espaces plus ou moins urbains, partageant un certain nombre de caractéristiques, ces dernières n'ont pas été mises en place volontairement par les acteurs. Il existe bien une relation entre les activités sociales et l'espace urbain, mais cette relation est dialectique (urbanisation ou processus de fabrique urbaine) et non pas causale (urbanisme). Je propose d'examiner comment la matérialité de la ville a pu enregistrer et être en partie conditionnée par le fonctionnement social de ses habitants, mais aussi comment cette même matérialité spatiale a pu influencer en retour les activités sociales.

\section{LE CONCEPT DE FABRIQUE URBAINE}

Suite au développement de l'archéologie médiévale et de la géographie urbaine, notamment en Grande-Bretagne ${ }^{1}$, les historiens intègrent de plus en plus la dimension spatiale et environnementale du développement des villes. L'espace urbain constitue désormais un chantier majeur de l'histoire urbaine, ce dont témoignent deux récentes publications de synthèse ${ }^{2}$. Ma propre recherche doctorale, dont une partie des résultats est présentée ici, s’inscrit dans ce courant

BIDDle M., «The towns », dans WiLson D. M. (éd.), The Archaelogy of Anglo-saxon England, Londres, 1976, pp. 99-150 ; BARKE M., "Morphogenesis, fringe-belts and urban size : an explotary essay », dans SLATER T. R. (éd.), The Built Form of Western Cities. Essays for M. R. G. Conzen on the occasion of his eightieth birthday, LeicesterLondres, Leicester University Press, 1990, pp. 279-99.

Palliser D. M. (éd.), The Cambridge Urban History of Britain, 1 (600-1540), Cambridge, Cambridge University Press, 2000, chapitre 8 «Topography of towns 600-1300»; BOUCHERON P., MENJOT D. et BOONE M., «La ville médiévale ", dans PINOL J.-L. (dir.), Histoire de l'Europe urbaine, 1, Paris, Éditions du Seuil, 2003, pp. 285592 , chapitre $5:$ «L'organisation du tissu urbain ». 
Noizet, Hélène, "La fabrique urbaine de Tours : une analyse dialectique entre sociétés et espaces urbains (IXe-XIII" siècle)", dans V oisinages, coexistences, appropriations. Groupes sociaux et territoires urbains (Moyen Âge - 16 siècle), édité par Chloé Deligne et Claire Billen, p. 19-37, Turnhout, Brepols, 2007.

historiographique ${ }^{3}$. Nous entrerons donc en ville par l'espace, avec le concept de fabrique urbaine, formalisé par H. Galinié à partir de la notion d'urban fabric, courante dans l'archéologie britannique 4 . Nous désignons par «fabrique urbaine» l'interaction permanente et dialectique de la structure de l'espace urbain avec l'activité sociale multiforme. Le cas de la ville de Tours constitue un bon exemple permettant de comprendre l'utilité de ce concept. La zone d'étude choisie couvre les parties occidentale et centrale de la ville comprise à l'intérieur de l'enceinte médiévale du XIVe siècle (plan 1), c'est-à-dire les quartiers de Saint-Martin et de Saint-Julien. Développée sur la rive gauche de la Loire, la ville de Tours comprend, à l'est la Cité, avec la cathédrale et l'enceinte du IVe siècle, à l'ouest le quartier de Saint-Martin, avec la basilique, protégée par un castrum construit au début du $\mathrm{X}^{\mathrm{e}}$ siècle et au nord duquel s'est développé Châteauneuf, et, entre les deux, une zone où a été reconstruit vers 940 le monastère de Saint-Julien, détruit par le raid viking de 853. Notre propos comprend trois parties : tout d'abord, l'analyse morphologique de l'espace urbain, puis l'analyse dialectique des relations socio-spatiales dans le quartier de Châteauneuf, et enfin une modélisation de la relation à la ville des chanoines et des moines, grâce à la méthode idéaltypique de Max Weber.

\section{ANALYSE MORPHOLOGIQUE DE L'ESPACE TOURANGEAU}

La structuration de l'espace urbain médiéval est un processus de longue durée, qui couvre plusieurs siècles. Le premier plan parcellaire suffisamment précis pour permettre une description n'est autre que le cadastre dit napoléonien, levé à Tours à partir de 1836 (plan 1). Cependant, l'utilisation d'un document si tardif ne constitue pas un obstacle majeur car l'urbanisation de l'époque moderne ne fait qu'étendre et prolonger les tendances perceptibles dès le haut Moyen Âge ${ }^{5}$. L'échelle de temps qui est ici mise en œuvre permet d'utiliser un document subcontemporain: nous n'allons pas nous situer uniquement à l'échelle de la vie humaine, mais examiner comment cette échelle du projet s'articule avec une échelle de temps plus longue, celle de la fabrique de la ville. Il en est de même de l'échelle spatiale: il ne s'agit pas de mettre en relation telle parcelle précise avec une documentation écrite médiévale ou moderne, mais d'étudier la physionomie générale d'un grand ensemble de parcelles, qui forment des masses à l'échelle de la ville. Pour cela, l'espace urbain est considéré comme une source à part entière, dont on peut décrire la desserte (le réseau viaire), les îlots et les parcelles. L'analyse morphologique menée sur ce plan cadastral permet d'identifier trois zones différentes: le quartier de Saint-Julien, le quartier canonial de Saint-Martin et Châteauneuf (plan 2). Les calculs présentés ci-dessous ont été réalisés grâce à la

\footnotetext{
NoIZET H., La fabrique de la ville. Espaces et sociétés à Tours (IX ${ }^{e}-X I I I^{e}$ s.), Paris, Publications de la Sorbonne, 2007. GALINIÉ H., Ville, espace urbain et archéologie, coll. Sciences de la ville, 16, Tours, Maison des Sciences de la Ville, 2000.

La seule différence majeure concerne le percement de la rue Royale (aujourd'hui rue Nationale), orientée nordsud, qui élargit et connecte entre elles d'anciennes petites rues (plan 1). Cette opération de la fin du XVIII siècle comprenait également la construction d'un nouveau pont sur la Loire, dans le prolongement de la rue Royale et, sur l'autre rive, le percement d'une route, appelée aujourd'hui encore la Tranchée.
} 
Noizet, Hélène, "La fabrique urbaine de Tours : une analyse dialectique entre sociétés et espaces urbains (IXe-XIII ${ }^{\mathrm{e}}$ siècle)", dans Voisinages, coexistences, appropriations. Groupes sociaux et territoires urbains (Moyen Âge - 16e siècle), édité par Chloé Deligne et Claire Billen, p. 19-37, Turnhout, Brepols, 2007.

numérisation de ce cadastre, qui constitue un des niveaux du SIG de l'équipe Archéologie et Territoires ${ }^{6}$.

\section{1. Saint-Julien}

Puisque l'espace est considéré comme une source, il convient de citer nos sources (cf plan 3).

Le nombre de rues est assez faible au regard de la superficie de cette zone : au total, moins d'une dizaines de rues desservent 20 ha. Notons également une absence quasi-totale de place ou d'espace ouvert. On observe également la très forte prégnance des deux axes parallèles à la Loire, orientés est-ouest (l'axe rue du Commerce-rue Colbert et l'axe rue de la Scellerie-rue des Halles) : toutes les autres rues, qui sont orientées nord-sud et qui paraissent secondaires ${ }^{7}$, ont été déterminées par ces deux axes dont l'orientation résulte de l'influence structurante de la Loire, le long de laquelle la ville antique, Caesarodunum, s'est développée depuis le Haut Empire.

Les $9 / 10^{e}$ des îlots sont de grande taille et de forme quadrangulaire. Les 619 parcelles comprises dans ces 10 îlots couvrent au total une superficie de 17,02 ha: en moyenne, un îlot de cet espace mesure environ 1,7 ha et comporte 62 parcelles. La superficie moyenne d'une parcelle est de $270 \mathrm{~m}^{2}$. Ces moyennes masquent de profondes disparités entre l'intérieur d'un îlot et ses franges. Prenons l'exemple de l'îlot situé entre les rues Marceau, des Halles, Nationale et du Commerce : les 83 parcelles situées en front de rue ont une superficie totale de 0,959 ha, ce qui donne une superficie parcellaire moyenne de $120 \mathrm{~m}^{2}$. Au contraire, les parcelles à l'intérieur du l'îlot sont beaucoup moins nombreuses, mais bien plus vastes : nous pouvons y dénombrer 19 parcelles, avec une superficie totale de 1,652 ha. Ainsi, la superficie moyenne de la parcelle équivaut à $870 \mathrm{~m}^{2}$, soit 7 fois plus que la surface moyenne d'une parcelle située sur le front de rue (plan 4).

$\mathrm{Au}$ centre des îlots, les parcelles de grandes dimensions ont de formes variées - grossièrement rectangulaires ou en forme de ' $T$ ' ou de ' $L$ ' - et sont bordées tout autour par les petites parcelles en forme de lanières. La façade sur la rue de ces parcelles oblongues est presque toujours beaucoup plus courte que la longueur de la parcelle qui s'enfonce à l'intérieur de l'îlot. Certaines d'entre elles sont extrêmement fines et longues et font la jonction entre la rue et une parcelle enclavée à l'intérieur de l'îlot : il s'agit manifestement de passages aménagés pour accéder aux parcelles situées en son centre. Ce parcellaire laniéré est beaucoup plus dense le long des deux grandes artères parallèles à la Loire.

GALINIÉ H. et RODIER X., «TOTOPI : Topographie de Tours Pré-Industriel, un outil d'analyse urbaine », Les petits cabiers d'Anatole, 11, disponible sur www.univ-tours.fr/lat/Pages/F2htm.

Avant la fin du XVIII e siècle, il faut imaginer à la place de la large rue Nationale une série de petites rues. 
Noizet, Hélène, "La fabrique urbaine de Tours : une analyse dialectique entre sociétés et espaces urbains (IXe-XIII" siècle)", dans V oisinages, coexistences, appropriations. Groupes sociaux et territoires urbains (Moyen Âge - 16 siècle), édité par Chloé Deligne et Claire Billen, p. 19-37, Turnhout, Brepols, 2007.

\section{2. Le cloître de Saint-Martin}

Concernant le réseau viaire, la forme en peigne des rues Baleschoux, Rapin et Jules-Le Roy, est datable de la construction du castrum de Saint-Martin achevée en 918 : cette ligne parallèle au tracé de la fortification, qui répète la forme de l'enceinte (plan 1), dessert le rempart du côté intérieur. La rue est-ouest, qui longeait la basilique Saint-Martin ${ }^{8}$, coupait le castrum en deux moitiés : deux axes perpendiculaires à cette rue est-ouest (rue du Change et rue Descartes) divisent donc chaque moitié en quadrant. Le réseau de rues révèle une organisation spatiale volontaire, datable du début $\mathrm{X}^{\mathrm{e}}$ siècle?.

Les 8 îlots qui couvrent au total 5,836 ha, contiennent 153 parcelles : en moyenne, un îlot couvre environ 0,73 ha et comprend 19 parcelles (plan 5). Avec une superficie moyenne de $380 \mathrm{~m}^{2}$, les parcelles ont des dimensions assez grandes, sans être aussi importantes qu'à l'intérieur des îlots du groupe précédent. Les formes de ces îlots sont plus variées qu'à Saint-Julien: si certains sont quadrangulaires, d'autres présentent une forme en ' $L$ ' ou comportent des excroissances. Il existe de plus une régularité à l'intérieur des îlots, étant donné que les parcelles sont toutes quadrangulaires et qu'il n'y a pas ici de disparité entre l'intérieur et la bordure des îlots.

\section{3. Châteauneuf}

Ce troisième ensemble dispose d'un réseau viaire beaucoup plus étoffé et complexe, que ce soit du point de vue du nombre des voies ou de la variété des orientations (plan 6). Il comporte aussi le plus grand nombre de places (places du Grand-Marché, de Châteauneuf, place Plumereau...). L'orientation vers la Loire est un autre élément remarquable, notamment pour la partie septentrionale de Châteauneuf, qui correspond au suburbium du castrum de Saint-Martin. Cette disposition, qui n'a pas d'équivalent à Tours, révèle un tropisme exercé par le fleuve $^{10}$ : toutes les rues sont en effet orientées de manière à permettre la circulation entre la Loire et le suburbium et le castrum de Saint-Martin.

Il apparaît clairement que le nombre des îlots, 51, est beaucoup plus important que dans les deux autres ensembles et que leur taille est beaucoup plus petite. Le nombre des parcelles paraît singulièrement élevé comparé aux deux précédents : 1094 parcelles pour Châteauneuf, contre 153 dans le cloître et 619 à Saint-Julien. Pourtant, ces parcelles ne couvrent que de 10,997 ha: pour un seul hectare, Châteauneuf comprend 99 parcelles, tandis que le cloître n'en contient que 26 et le secteur de Saint-Julien 36.

\footnotetext{
La basilique a été détruite au début du XIXe siècle et la rue des Halles prolongée dans la nef de la basilique : le carrefour visible sur le cadastre napoléonien correspond au chœur de la basilique.

Galinié H., "Genèse du paysage urbain », dans Chevalier B. (éd.), Histoire de Tours, Toulouse, Privat, 1985, pp. 11-48.

10 Galinié H. et Rodier X., "Les modifications du trait de la rive gauche de la Loire dans l'ouest de Tours », dans CARCAUd N. (coord.), Géoarchéologie de la Loire moyenne, Résultats 1996-1999, 2001, pp. 29-36, article disponible sur le site http://zal.brgm.fr, SRA Région Centre.
} 
Noizet, Hélène, "La fabrique urbaine de Tours : une analyse dialectique entre sociétés et espaces urbains (IXe-XIII ${ }^{\mathrm{e}}$ siècle)", dans Voisinages, coexistences, appropriations. Groupes sociaux et territoires urbains (Moyen Âge - 16 siècle), édité par Chloé Deligne et Claire Billen, p. 19-37, Turnhout, Brepols, 2007.

À Châteauneuf, un îlot couvre en moyenne 0,21 ha et comporte 21 parcelles. La superficie parcellaire moyenne y est des plus réduites, soit $100 \mathrm{~m}^{2}$ : ainsi, ces parcelles sont presque 4 fois plus petites que dans le cloître, et presque 9 fois plus petites qu'à l'intérieur des îlots de Saint-Julien. Ces parcelles de Châteauneuf ont en revanche une taille proche de celles des fronts de rue bordant les îlots de Saint-Julien. Les formes des îlots sont également beaucoup plus variées qu'ailleurs dans la ville : îlots rectangulaires très allongés dans le sens nord-sud ou est-ouest, formes curvilignes, formes comportant de nombreux décrochements et retours, qui donnent un aspect labyrinthique au réseau viaire. Nous constatons également qu'à l'intérieur des îlots de Châteauneuf, les parcelles sont disposées dosà-dos, sans qu'il y ait de parcelles intermédiaires, enclavées au milieu des îlots, comme à Saint-Julien. Ainsi, les caractéristiques majeures de cette zone sont une très forte densité et une très forte fragmentation du parcellaire, auxquelles s'ajoute une grande diversité des formes des îlots et des parcelles. Il s'agit donc d'un véritable espace urbain, au sens du géographe J. Lévy ${ }^{11}$. Ce dernier définit la ville comme une situation spatiale caractérisée par la concentration d'une société en un lieu en sorte d'y maximiser la densité et la diversité des interactions sociales. La ville est en effet une des trois possibilités que les sociétés ont pour lutter contre la distance, les deux autres étant la mobilité matérielle (les transports) et la mobilité immatérielle (le courrier et les télécommunications). J. Lévy précise ainsi que «pour relier deux objets sociaux $a$ et $b$, soit on déplace matériellement $a$ vers $b$ (mobilité), soit on se contente de faire circuler de l'information de $a$ vers $b$ (télécommunication), soit enfin on place $a$ et $b$ en contact direct, de manière à éliminer la distance en créant un lieu (co-présence) ».

\section{4. Des pratiques socio-spatiales}

À ces types d'espaces correspondent des pratiques sociales spécifiques. Le secteur de Saint-Julien témoigne de la durabilité des conséquences de l'utilisation agricole de ces terres : les grandes parcelles au centre des îlots sont issues des pièces de culture, tandis que les franges de ces îlots ont été subdivisées en un parcellaire laniéré et dense, pour construire des maisons, dont les façades donnaient sur la rue. Il a été nécessaire par la suite d'aménager des petits passages pour accéder aux terres arables, champs et vignes, situées au centre de l'îlot, comme en témoigne les parcelles extrêmement étirées en longueur. Cette utilisation agricole explique aussi que l'on n'ait pas de réseau viaire très développé, puisqu'une zone de culture n'a pas besoin d'une desserte très étoffée. Le cloître de Saint-Martin, au sens de quartier canonial, est une zone résidentielle de type élitaire: ces parcelles plus ou moins carrées correspondent à l'époque moderne à des hôtels urbains. Ces hôtels, qui comportent des jardins en fonds de parcelles, sont réservés aux chanoines ainsi qu’à l'élite urbaine. Cette fonction résidentielle explique que les réseaux viaires soient un peu plus denses que dans la zone agricole pour accéder à ces vastes propriétés urbaines, tout en restant assez faibles, dans la mesure où la densité de population

11 LÉvy J., Le tournant géographique. Penser l'espace pour lire le monde, coll. Mappemonde, Paris, Belin, 1999, pp. 16-19 et p. 199, et plus globalement les chapitres 7 à 10, pp. 195-268. 
Noizet, Hélène, "La fabrique urbaine de Tours : une analyse dialectique entre sociétés et espaces urbains (IXe-XIII" siècle)", dans Voisinages, coexistences, appropriations. Groupes sociaux et territoires urbains (Moyen Âge - 16 siècle), édité par Chloé Deligne et Claire Billen, p. 19-37, Turnhout, Brepols, 2007.

reste peu élevée en raison de la ségrégation sociale. Au contraire, Châteauneuf est caractéristique des espaces commerciaux et artisanaux, avec de nombreuses ateliers, boutiques ou petites habitations, toutes donnant sur la rue, qui expliquent l'intensité de la fragmentation et de la densité du parcellaire. La pression foncière y est stimulée par une forte densité démographique et par la concentration des activités commerciales. Le passage est donc très important: d'où la nécessité de nombreuses rues et de places de marché. Le tropisme de la Loire s'explique également par cette prédominance du secteur productif et commercial, puisque la Loire était une voie d'échanges primordiale.

Les diagrammes réalisés à partir du tableau récapitulatif ci-dessous (tableau 1 et diagramme 1) montrent à quel point cette partie de la ville se singularise par rapport aux deux précédentes :

\begin{tabular}{|l|c|c|c|}
\hline & Saint-Julien & Saint-Martin & Châteauneuf \\
\hline Nb d'îlots (unités) & 10 & 8 & 51 \\
Nb de parcelles (unités) & 619 & 153 & 1094 \\
\hline Surface moy./îlot (ares) & 170 & 73 & 21 \\
Surface moy./parcelle $\left(\mathrm{m}^{2}\right)$ & 270 & 380 & 100 \\
\hline Superficie tot. du secteur & 17,02 & 5,836 & 10,997 \\
Parcelles/hectare & $\mathbf{3 6 , 4}$ & $\mathbf{2 6 , 2}$ & $\mathbf{9 9 , 5}$ \\
\hline
\end{tabular}

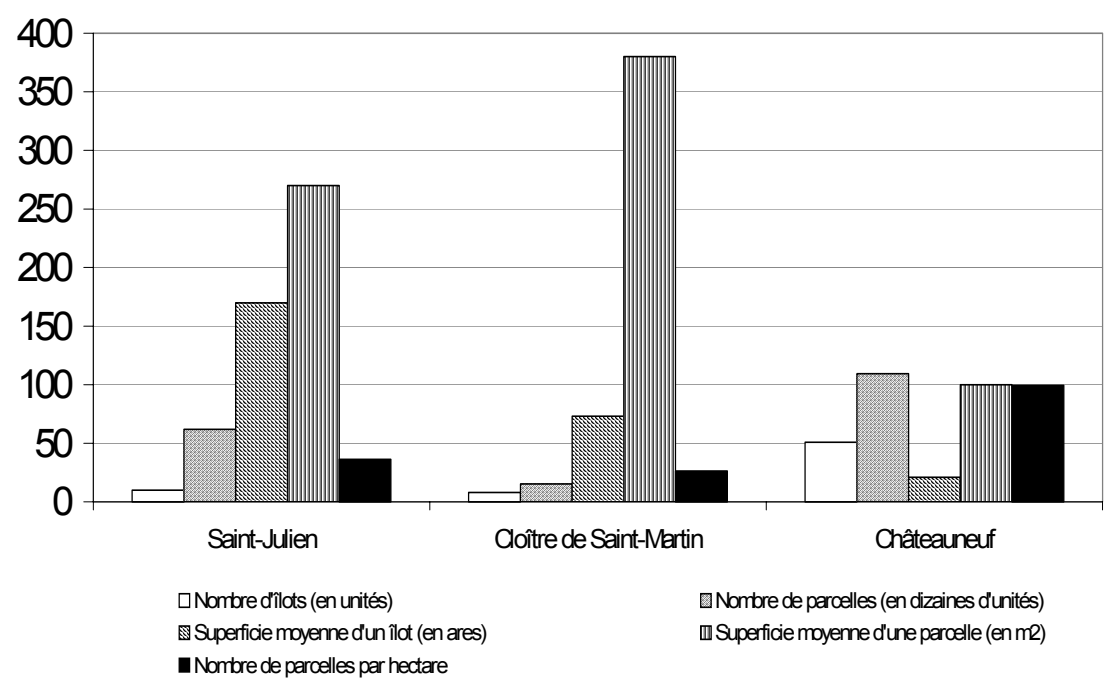

Tableau 1 et diagramme 1: comparaison des profils spatiaux dans trois quartiers tourangeaux

Autant les diagrammes de Saint-Julien et du cloître de Saint-Martin ont une allure similaire, avec un nombre relativement restreint d'îlots et de parcelles, qui ont en revanche des tailles assez grandes, autant celui de Châteauneuf dispose d'un très grand nombre d'îlots et de parcelles, dont les dimensions sont à l'inverse très 
Noizet, Hélène, "La fabrique urbaine de Tours : une analyse dialectique entre sociétés et espaces urbains (IXe-XIII ${ }^{\mathrm{e}}$ siècle)", dans Voisinages, coexistences, appropriations. Groupes sociaux et territoires urbains (Moyen Âge - 16e siècle), édité par Chloé Deligne et Claire Billen, p. 19-37, Turnhout, Brepols, 2007.

réduites. Il est donc possible de ramener ces trois quartiers à deux types spatiaux, seul celui de Châteauneuf étant véritablement un type urbain.

La question que nous posons maintenant est simple : comment en est-on arrivé là ? Comment expliquer l'existence de ces différents quartiers ?

\section{ANALYSE DIALECTIQUE DE RELATIONS SOCIO-SPATIALES}

Un seul projet volontaire a pu être identifié : le réseau viaire du quartier canonial, en relation avec la construction du castrum de Saint-Martin au début du $\mathrm{X}^{\mathrm{e}}$ siècle. Mais, pour toutes les autres caractéristiques spatiales décrites ci-dessus, on ne trouve, dans les sources écrites, aucune trace d'une quelconque planification: aucune personne, ni aucun groupe social n'a formulé le projet de construire ces espaces, d'organiser la matérialité de la ville de cette manière ou de telle autre, et encore moins de mettre en place une ville. Même chez les bourgeois, dont le mouvement communal est traditionnellement considéré comme le mouvement précurseur présidant à la formation d'une ville, il n'y a aucune trace d'une volonté de constituer physiquement une ville. Les bourgeois ne pensaient pas, en obtenant des chartes et des franchises, "créer une ville»: simplement, ils cherchaient à s'autonomiser du pouvoir seigneurial et à régler eux-mêmes leurs affaires.

Pourtant, ces différents types d'espaces existent bel et bien. Il faut admettre qu'il n'y a pas de causalité directe et immédiate à l'urbanisation: on ne peut déterminer un fait, un événement ou un acteur social qui serait fondateur. Le paradoxe paraît insurmontable dès lors que l'on se limite à l'échelle de la vie humaine et que l'on raisonne en terme de projet. Puisqu'il n'est pas possible de rendre compte de la structuration de l'espace urbain en la considérant au niveau de l'intention ou de la finalité des acteurs, il faut quitter le registre de la compréhension pour entrer dans celui de l'explication. Je propose donc de mettre en relation les organisations parcellaires décrites ci-dessus avec les activités sociales opérantes, c'est-à-dire les configurations sociales qui participent à expliquer la structure urbaine de cet espace, sans qu'aucune finalité urbaine n'ait été préconçue. Nous allons donc vérifier comment, en diachronie, les différents comportements des acteurs ont indirectement favorisé la mise en place d'une structure plus ou moins urbaine, ainsi que les effets en retour de cette structure sur les configurations sociales observées. Pour des raisons de place, je me limiterai ici à l'analyse du secteur de Châteauneuf, mais le même raisonnement pourrait être tenu à Saint-Julien ou dans le cloître.

À l'inverse des espaces de Saint-Julien et du cloître, l'urbanisation a été très forte à Châteauneuf. Il s'y est produit, depuis le IX ${ }^{\mathrm{e}}$ siècle, une succession d'activités sociales, qui tendaient, toujours de manière indirecte et involontaire, à renforcer la densité et la diversité du parcellaire. Les six configurations présentées ci-dessous répondaient à chaque fois à un problème spécifique, qui n'avait en soi rien d'urbain. Cependant, si aucun projet urbain n'a été en tant que tel formulé, toutes ces situations ont favorisé la structuration urbaine de cet espace.

Le premier moment se situe entre 816 et 862 : il correspond à l'adoption de la règle canoniale à Saint-Martin, ainsi qu'à la mise en place d'une organisation 
Noizet, Hélène, "La fabrique urbaine de Tours : une analyse dialectique entre sociétés et espaces urbains (IX"-XIII" siècle)", dans Voisinages, coexistences, appropriations. Groupes sociaux et territoires urbains (Moyen Âge - 16 siècle), édité par Chloé Deligne et Claire Billen, p. 19-37, Turnhout, Brepols, 2007.

spécifique des ressources destinées à répondre aux besoins de consommation ${ }^{12}$. Le choix de la règle canoniale influe de deux manières sur le fonctionnement de cet établissement. Tout d'abord, le régime de vie, et notamment alimentaire, des chanoines est très confortable dans la mesure où il ne comporte pas d'interdiction particulière : ainsi la viande est autorisée, ce qui implique de disposer des structures de ravitaillement adaptées à ce type de denrée périssable. De plus, aucun travail manuel n'est exigé des chanoines : ceux-ci doivent donc s'en remettre totalement à une main-d'œuvre externe, chargée d'assurer la totalité des travaux et des tâches quotidiennes indispensables au bon fonctionnement d'une telle communauté. Cette configuration canoniale nécessite donc une domesticité nombreuse, ainsi que des produits en grande quantité et variés, avec notamment de la viande. Il faut ajouter à cela le nombre très élevé de chanoines appartenant à l'institution tourangelle: pas moins de 200 chanoines vivent à cette époque à Saint-Martin, ce qui en fait localement la communauté ecclésiastique la plus importante du point de vue de l'effectif. Enfin, le dernier élément qui entre en jeu dans cette configuration initiale est le pèlerinage de Saint-Martin. La localisation de la tombe de saint Martin, dans une nécropole publique à $1500 \mathrm{~m}$ à l'ouest de la cathédrale, correspondrait en réalité à un des premiers moments de l'histoire urbaine post-romaine de cette partie de la ville, moment qu'il conviendrait de mettre en exergue dans le cas d'une étude qui inclurait l'Antiquité tardive. La présence de la tombe de saint Martin s'est révélé être une véritable mine d'or, dans la mesure où les visites des nombreux pèlerins fournissaient des revenus à la population locale qui leur proposait des services. Rappelons que, au Moyen Âge, le pèlerinage à Saint-Martin de Tours était le quatrième en importance en Europe, après Saint-Jacques de Compostelle, Jérusalem et Rome. On comprend dès lors que les besoins de consommation fussent très élevés : il fallait donc beaucoup de produits et beaucoup d'hommes pour assurer « la bonne gestion de la maisonnée », qui constituait, selon J.-P. Devroey ${ }^{13}$, l'objectif premier de ces propriétaires ecclésiastiques.

Les effets induits par la présence d'une telle communauté expliquent donc la mise en place d'un parcellaire dense et diversifié dès le haut Moyen Âge, et ce, même si les chanoines n'ont à aucun moment voulu construire une ville. La structuration véritablement urbaine de l'espace a été impulsée par l'obligation qu'il y avait de loger et faire vivre toute cette population cléricale et laïque : nous avons calculé que l'effectif des habitants à Saint-Martin pourrait se situer, à l'époque carolingienne, dans un ordre de grandeur d'environ 1500 à 2000 personnes $^{14}$, effectif auquel il convient d'ajouter la population passagère des pèlerins. Les chanoines ont organisé leur patrimoine de manière à faire face à de tels besoins. Pour cela, ils ont mobilisé les compétences géographiques et fluviales de leurs possessions, dont certaines ont été mises en réseau. Or la mise en œuvre de ces ressources a conditionné une caractéristique importante de l'espace urbain : l'attraction exercée par la Loire. Il semble que le chapitre ait fait parvenir par bateau certains des

12 Noizet H., «Le centre canonial de Saint-Martin de Tours et ses domaines périphériques en Val de Loire (IX ${ }^{\mathrm{e}}$ $\mathrm{X}^{\mathrm{e}}$ siècles)», Annales de Bretagne et des Pays de l'Ouest, 2002, 109, 2, pp. 14-37.

13 Devroey J.-P., «'Ad utilitatem monasterii'. Mobiles et préoccupations de gestion dans l'économie monastique du monde franc (VIII ${ }^{\mathrm{e}}-\mathrm{IX}{ }^{\mathrm{e}}$ siècles) », Revue bénédictine, 1993, 103, pp. 224-40, ici, pp. 239-241.

14 Noizet H., «Le centre canonial de Saint-Martin... », art.cit. 
Noizet, Hélène, "La fabrique urbaine de Tours : une analyse dialectique entre sociétés et espaces urbains (IXe-XIII ${ }^{\mathrm{e}}$ siècle)", dans Voisinages, coexistences, appropriations. Groupes sociaux et territoires urbains (Moyen Âge - 16e siècle), édité par Chloé Deligne et Claire Billen, p. 19-37, Turnhout, Brepols, 2007.

produits indispensables à son fonctionnement, comme la laine. Dès 816, il avait obtenu à cet effet les exemptions de taxes nécessaires à de tels échanges, et tout particulièrement pour la Loire et ses affluents, sur lesquels douze de leurs bateaux pouvaient circuler librement. Il est donc logique qu'à Tours, la Loire conditionne fortement l'organisation du réseau viaire, puisque c'est de là que provenaient une grande partie des produits utiles au fonctionnement de l'agglomération martinienne.

Les dispositions, prises par les chanoines pour assurer leur ravitaillement en viande, ont favorisé d'une certaine manière la densification du parcellaire. Ceux-ci ont fait le choix d'installer les structures d'élevage à environ trois kilomètres au sud de la Loire, dans les prairies des Varennes, c'est-à-dire non loin, mais pas sur le site de Tours : ces installations de type agricole ont donc été rejetées à une certaine distance du quartier de Saint-Martin. Les choses auraient été différentes si les chanoines avaient décidé de mettre en place leur centre d'élevage dans l'environnement immédiat de leur basilique, à Tours même. Une grande partie du sol aurait alors été gelée par ces terrains laissés à l'état de prés, bloquant ainsi le développement d'habitations à proximité de la basilique. Cette configuration, qui dissociait lieu de production et lieu de consommation, a donc indirectement favorisé l'urbanisation de cet espace. Comme pour la Loire, il n'y a aucun projet urbain spécifique, mais simplement la volonté d'exploiter au mieux les potentialités de leur environnement, à savoir les prairies des Varennes, qui constituaient des terres d'embouche enrichies par les limons, déposés lors des inondations fréquentes dans cette dépression topographique. Si l'on peut souligner la capacité de ces hommes à s'insérer dans leur environnement physique, à la fois terrestre et fluvial, leur dessein n’était pas de créer à proprement parler une ville.

Cette configuration initiale fut l'objet d'un ré-ordonnancement, au début du $\mathrm{X}^{\mathrm{e}}$ siècle, en liaison avec la construction du castrum de Saint-Martin ${ }^{15}$. La nouvelle modalité de l'accord entre chanoines et habitants laïcs s'est traduite par l'installation des laïcs dans la moitié nord du castrum, tandis que la moitié sud était destinée en théorie aux seuls chanoines. Une autre possibilité aurait consisté à exclure les laïcs de la totalité du castrum, comme les moines de Saint-Julien l'ont fait avec leur enclos. Or ce n'est pas ce choix qui a été fait par les chanoines. La raison est certainement à chercher du côté de leur insertion dans le siècle: le mode de vie canonial n'impliquait pas en effet une distance entre clercs et laïcs aussi stricte que dans un monastère bénédictin. Cette dévolution de la partie septentrionale du castrum aux seuls laïcs a joué un rôle majeur dans la fabrique urbaine. Elle constitue le premier facteur expliquant deux de ses caractéristiques structurelles : le fait tout d'abord que le parcellaire soit très différent entre le nord et le sud du castrum (plan 7), et, à l'inverse, le fait qu'il soit de même nature dans cette moitié nord et dans le bourg Saint-Pierre-le-Puellier.

Le tropisme de la Loire fut également conforté par cette nouvelle configuration de 918. Du point de vue de la structure urbaine, l'accès à la Loire a réactivé l'orientation du réseau viaire. En 918 et 919, la communication avec la Loire fut manifestement recherchée comme en témoignent les deux temps de la

15 Noizet H., Fabrique de la ville..., op. cit., chapitre 5, pp. 97-119. 
Noizet, Hélène, "La fabrique urbaine de Tours : une analyse dialectique entre sociétés et espaces urbains (IX"-XIII" siècle)", dans Voisinages, coexistences, appropriations. Groupes sociaux et territoires urbains (Moyen Âge - 16 siècle), édité par Chloé Deligne et Claire Billen, p. 19-37, Turnhout, Brepols, 2007.

concession de l'immunité pour le suburbium du castrum de Saint-Martin ${ }^{16}$ : d'abord concédée pour le seul castrum, l'immunité fut ensuite étendue à toute la bande de terrain comprise entre la Loire et le castrum nouvellement construit. Une telle mise en place de la nouvelle unité juridique autour de Saint-Martin montre que la Loire restait une préoccupation majeure: elle était toujours un axe de communication essentiel entre Saint-Martin de Tours et le reste du monde. Une autre preuve en est la localisation de la boulangerie en 920 : à cette date, elle se trouve dans le bourg de Saint-Pierre-le-Puellier, justement entre le castrum et la Loire. Cette localisation, dont on ne sait si elle est nouvelle ou si elle préexiste au castrum, prouve que les structures économiques nécessaires aux chanoines n'étaient pas cantonnées au quartier canonial. Celles-ci auraient pu en effet se situer dans la moitié sud du castrum, puisque c'est là que les chanoines sont censés vivre. Cette localisation aurait été d'autant plus justifiée que Tours avait souffert des incursions scandinaves durant la seconde moitié du IX ${ }^{\text {e }}$ siècle: or les Vikings étaient justement arrivés par bateau depuis la Loire. Ainsi, le traumatisme viking n'a pas modifié l'organisation de l'espace à Tours : l'accès à la Loire restait une priorité pour les chanoines.

Un troisième moment important paraît correspondre au premier quart du $\mathrm{XI}^{\mathrm{e}}$ siècle, lors du transfert des moniales de Notre-Dame-de-l'Écrignole (plan 1) à Beaumont ${ }^{17}$. Le trésorier Hervé a profité de l'énorme chantier de la reconstruction de la basilique pour éloigner ces bénédictines, qui se trouvaient juste au nord de l'église capitulaire. Il cherchait à ce qu'elles soient mieux installées, dans un endroit qui présente les qualités de calme et de confort requis pour ce type de communauté féminine: c'est pourquoi elles quittèrent l'area magna (place de Châteauneuf) pour Beaumont, c'est-à-dire la campagne, dans la périphérie sud-ouest de l'agglomération martinienne. Cette place de Châteauneuf constituait en effet un environnement peu propice à la vie bénédictine de ces moniales, dans la mesure où elle était fréquentée par les marchands et visiteurs de toutes sortes : l'activité y était singulièrement dense et variée en raison de la proximité de cette place avec le tombeau de saint Martin. Autrement dit, l'espace urbain hérité des $\mathrm{IX}^{\mathrm{e}}-\mathrm{X}^{\mathrm{e}}$ siècles explique en partie la décision d'Hervé. Mais, en retour, cet accord entre le trésorier et les moniales a favorisé à nouveau la densification et la diversification du parcellaire : en effet, jusque là la communauté de Beaumont bloquait par son emprise une partie du sol de cette place. Leur départ a libéré du terrain qui a pu ensuite être bâti et densément occupé. Désormais, il devenait possible d'aménager, à proximité ou à la place des bâtiments monastiques, plusieurs maisons ou boutiques, permettant à un plus grand nombre de personnes de vivre et d'exercer leurs activités autour de cette place. L'expulsion de ces moniales a ainsi favorisé l'urbanisation dans la moitié nord du castrum.

Puis, peu après, vers le milieu du XIe siècle, les sources nous donnent à voir une nouvelle situation, liée cette fois-ci au bourg de Saint-Pierre-le-Puellier. Les serviteurs laïcs de Saint-Julien ont été rejetés de l'environnement immédiat du

16 Lauer P. (éd.), Recueil des actes de Charles III le Simple, roi de France (893-923), 2 vol., Paris, 1940-1949, nº 98 (1er décembre 918) et $\mathrm{n}^{\circ} 101$ (27 juin 919). Pour s'assurer de leur sincérité, voir respectivement: DE FONT-RÉAULX J., "Les diplômes de Charles le Simple », Annales de l'Université de Grenoble, 1943, 19, pp. 29-49 ; NOIZET H., Pratiques spatiales..., op. cit., pp. 100-106.

17 NOIZET H., Fabrique de la ville..., op. cit., pp. 182-186. 
Noizet, Hélène, "La fabrique urbaine de Tours : une analyse dialectique entre sociétés et espaces urbains (IXe-XIII ${ }^{\mathrm{e}}$ siècle)", dans Voisinages, coexistences, appropriations. Groupes sociaux et territoires urbains (Moyen Âge - 16e siècle), édité par Chloé Deligne et Claire Billen, p. 19-37, Turnhout, Brepols, 2007.

monastère, pour être installés à proximité de Saint-Pierre-le-Puellier ${ }^{18}$. Ce choix, qui résulte tout d'abord de la volonté monastique d'éloigner les habitants laïcs de l'enclos de Saint-Julien, s'explique aussi par la préexistence du bourg de Saint-Pierrele-Puellier : il était commode d'installer à côté de ce bourg ces serviteurs, puisqu'il s'y trouvait déjà toutes les structures favorables au développement, c'est-à-dire l'espace en bordure de fleuve, mais également les structures économiques nécessaires à leurs obligations vis-à-vis des moines, à savoir des produits, des marchés, des ateliers... Ce faisant, ces hommes ont agrandi d'autant ce secteur. Là encore, l'effet d'entraînement a joué à plein : l'espace urbain a tout d'abord favorisé cette modalité de l'accord entre moines et laïcs de Saint-Julien, avant d'être stimulé à son tour par ce fonctionnement social.

Un cinquième moment peut être défini à la fin du XIe siècle, lorsque des banquiers et des orfèvres établissent leurs étaux sur la place de Châteauneuf, en accord avec les chanoines de Saint-Martin. Le désir de ces commerçants et artisans de développer leur activité les a poussés à choisir cette place. C'est bien parce qu'il y avait une demande économique forte à cet endroit que ces hommes s'y sont installés : la présence de nombreux pèlerins, encore accrue par l'augmentation des possibilités de logement et d'accueil à la suite du départ des moniales au début du $\mathrm{XI}$ e siècle, justifiait de proposer de nouveaux services de change et de ventes de produits d'artisanat. La structure urbaine a donc joué en faveur de cette nouvelle modalité de l'accord entre les chanoines et les habitants laïcs du castrum. Mais, par la suite, il est certain que l'installation de ces changeurs et artisans a intensifié d'autant l'activité urbaine et commerciale de cette place, entraînant la densification de la structure parcellaire. Cette nouvelle configuration sociale induit ainsi une augmentation de la pression foncière dans ce secteur.

Ces trois configurations du $\mathrm{XI}^{\mathrm{e}}$ siècle ont chacune leur contexte bien particulier - ici le respect de la clôture monastique féminine, là l'installation des hommes au service de Saint-Julien, ailleurs la recherche d'un profit économique mais elles ont toutes en commun d'interagir avec l'espace urbain. Elles partagent deux choses : le fait d'être en partie conditionnées par la structure matérielle de la ville et, en retour, le fait de renforcer le processus de densification et de diversification du parcellaire à Châteauneuf. Cette relation dialectique entre espace urbain et activité sociale, qui s'est singulièrement accélérée au XIe siècle, se prolonge encore au XII ${ }^{\mathrm{e}}$ siècle.

Au milieu du XII e siècle, un nouvel accord conclu entre les bourgeois, le roi et les chanoines constitue un autre moment important dans la longue chaîne des interactions entre l'espace urbain et l'activité sociale à Châteauneuf ${ }^{19}$. Les bourgeois acquièrent en effet certains privilèges qui leur permettent de développer plus librement leurs activités économiques, qu'il s'agisse de la vente du vin dans les tavernes ou le prêt d'argent à des taux fort rémunérateurs. Il est certain que si les bourgeois de Châteauneuf cherchent à obtenir ces concessions auprès du trésorier du chapitre, c'est parce qu'il y a un important potentiel de consommateurs, qui peut leur rapporter beaucoup. C'est l'existence a priori d'une forte densité urbaine qui

8 NoIzeT H., Fabrique de la ville..., op. cit., pp. 134-135.

19 NoIZET H., Fabrique de la ville..., op. cit., pp. 252-256. 
Noizet, Hélène, "La fabrique urbaine de Tours : une analyse dialectique entre sociétés et espaces urbains (IXe-XIII" siècle)", dans V oisinages, coexistences, appropriations. Groupes sociaux et territoires urbains (Moyen Âge - 16 siècle), édité par Chloé Deligne et Claire Billen, p. 19-37, Turnhout, Brepols, 2007.

justifie la démarche des bourgeois, dans la mesure ou ceux-ci cherchent à exploiter la présence d'une population à la fois nombreuse et variée, comme lors du concile de Tours de $1163^{20}$. Puis, là encore, cette modalité de l'accord entre chanoines et habitants de Châteauneuf réactive le processus de densification de la structure urbaine: l'ouverture des tavernes et des lieux d'accueil des visiteurs, qui a suivi l'octroi de ces privilèges, témoigne de la lä̈cisation de l'accueil des pèlerins et participe ainsi à accroître l'urbanisation dans cette partie de la ville.

Si je m'arrête ici, il est probable que la longue chaîne des relations dialectiques entre cet espace et les acteurs sociaux concernés se prolonge au-delà du $\mathrm{XII}$ e siècle : c'est ce processus que nous proposons d'appeler « fabrique urbaine ».

\section{MOINES ET CHANOINES : TYPES IDÉAUX ET URBANISATION}

Du côté de Saint-Julien et du cloître, la tendance inverse a été observée : là, les configurations socio-spatiales qui se sont succédé ont toutes freiné le développement véritablement urbain de ces secteurs. Comme l'espace de Châteauneuf s'est développé en relation avec le chapitre de Saint-Martin, et comme l'établissement de Saint-Julien est monastique, le rapprochement a été fait entre la règle de vie de ces communautés et leur rapport à l'espace urbain. Les effets de structure induits par le fonctionnement canonial ou monastique peuvent être formalisés grâce à l'outil méthodologique de Max Weber, l'idéal-type : il s'agit d'un outil construit abstraitement, qui établit toutes les caractéristiques possibles d'un phénomène, par exemple la ville, et qui, appliqué à une situation historique déterminée, par exemple à la ville du Moyen Âge, permet de déterminer en quoi celui-ci se singularise par rapport aux villes d'autres époques ou d'autres régions.

L'idéal-type est donc une construction qui n'existe pas en soi, mais qui sert à mettre en évidence des relations réelles et empiriques. Il est idéal, parce qu'il est une abstraction rationnelle et pure, qui ne correspond quasiment jamais aux phénomènes empiriques : le type idéal accentue les traits pour rendre plus intelligible le projet sous-jacent en train de se constituer. Cette méthode repose sur la position de principe selon laquelle il n'est pas possible de restituer l'extraordinaire complexité de la réalité : le fait que l'infinité du réel soit irréductible à un système de lois légitime la multiplicité des points de vue. Il faut garder à l'esprit que l'idéal-type n'est qu'un outil qui permet la pluralité des angles d'approche : il est à peu près le contraire d'une définition univoque. Cette méthode n'est utilisée que dans la perspective d'une problématique précise et bien définie: un idéal-type n'est pas une analyse en soi d'un objet, mais la simplification des traits d'un objet pour mettre en valeur son originalité par rapport à un processus ${ }^{21}$.

20 Le pape Alexandre III est venu à Tours, en 1163, pour y tenir un concile, le 19 mai. Les bourgeois avaient anticipé l'affluence des visiteurs et en ont profité pour augmenter les prix des logis destinés à recevoir cette masse de personnes. Devant les montants exigés par les hôteliers, qui atteignent apparemment des sommets, le roi Louis VII fixe un prix maximum de six livres, pour les logis les plus chers, les autres se payant à l'avenant: DeLisle L. (éd.), Recueil des historiens de Gaule et de France, Paris, 1874, 16, p. 47, n ${ }^{\circ}$ CLII

21 Weber M., La ville, Paris, Aubier, éd. allemande 1947, éd. française 1982, traduction P. FrITSCH ; FrITSCH P., "Type, idéaltype, stéréotype », dans BOURDin A. et HirSCHHORN M. (éds.), Figures de la ville: autour de Max Weber, Paris, Aubier, 1985, pp. 83-95. 
Noizet, Hélène, "La fabrique urbaine de Tours : une analyse dialectique entre sociétés et espaces urbains (IXe-XIII ${ }^{\mathrm{e}}$ siècle)", dans Voisinages, coexistences, appropriations. Groupes sociaux et territoires urbains (Moyen Âge - 16 siècle), édité par Chloé Deligne et Claire Billen, p. 19-37, Turnhout, Brepols, 2007.

À partir des sources normatives (règles canoniale et monastique), j’ai construit deux types idéaux des chanoines et des moines en fonction de cette problématique de l'urbanisation (tableau 2). Rappelons que ces types idéaux ne constituent pas une définition, encore moins une analyse précise des objets évoqués, mais qu'ils constituent des outils pour répondre à une question que l'on peut formuler ainsi : pourquoi certains espaces sont-ils devenus plus urbains que d'autres, c'est-à-dire plus denses et plus diversifiés ?

Ces deux types idéaux instituent un rapport au monde totalement différent et influent chacun à sa manière sur le processus de la fabrique urbaine. Ils rendent compte du fait que l'activité sociale induite par le fonctionnement canonial tend à produire un espace plus urbain que celui engendré par le fonctionnement monastique. Les spécificités canoniales favorisent en effet le processus de densification et la diversification de la société urbaine et de l'espace urbain. L'élément fondamental est la fonction assignée à chacune de ces communautés : si les chanoines sont là pour répondre directement aux besoins liturgiques et sacramentels de la population laïque, c'est de l'intérieur que les moines doivent tenter de sauver le monde. La démarche est donc radicalement différente.

\begin{tabular}{|c|c|c|c|c|c|c|}
\hline & Fonction & Aliments & Vêtements & $\begin{array}{c}\text { Travaux } \\
\text { d'entretien }\end{array}$ & Habitation & $\begin{array}{c}\text { Contact avec les } \\
\text { laïcs }\end{array}$ \\
\hline 1 & $\begin{array}{l}\text { assurer les } \\
\text { besoins } \\
\text { liturgiques/ } \\
\text { sacramentels } \\
\text { d'une population } \\
\text { laïque }\end{array}$ & $\begin{array}{l}\text { variés et } \\
\text { riches } \\
\text { (avec } \\
\text { viande) }\end{array}$ & $\begin{array}{l}\text { autorisation } \\
\text { de porter de la } \\
\text { laine et du lin ; } \\
\text { pas } \\
\text { d'interdiction } \\
\text { formelle }\end{array}$ & $\begin{array}{l}\text { aucun } \\
\text { travail } \\
\text { d'entretien } \\
\text { n'est } \\
\text { demandé }\end{array}$ & $\begin{array}{l}\text { maisons } \\
\text { personnel- } \\
\text { les dans le } \\
\text { quartier } \\
\text { canonial }\end{array}$ & $\begin{array}{l}\text { proximité } \\
\text { permanente } \\
\text { avec les laïcs ; la } \\
\text { seule réelle } \\
\text { interdiction } \\
\text { concerne la } \\
\text { présence des } \\
\text { femmes dans le } \\
\text { claustrum }\end{array}$ \\
\hline 2 & $\begin{array}{l}\text { sauver le monde } \\
\text { en atteignant la } \\
\text { perfection } \\
\text { évangélique par } \\
\text { la prière }\end{array}$ & $\begin{array}{l}\text { maigres et } \\
\text { limités } \\
\text { par des } \\
\text { interdits } \\
\text { (pas de } \\
\text { viande ; } \\
\text { jours de } \\
\text { jeûne) }\end{array}$ & $\begin{array}{l}\text { vêtements } \\
\text { médiocres; } \\
\text { interdiction de } \\
\text { porter de la } \\
\text { soie et des } \\
\text { peaux de } \\
\text { chèvre }\end{array}$ & $\begin{array}{l}\text { obligation } \\
\text { de } \\
\text { consacrer } \\
\text { plusieurs } \\
\text { heures par } \\
\text { jour au } \\
\text { travail } \\
\text { manuel }\end{array}$ & $\begin{array}{l}\text { dortoir } \\
\text { commun ou } \\
\text { cellules dans } \\
\text { un même } \\
\text { bâtiment à } \\
\text { l'intérieur } \\
\text { de l'enclos }\end{array}$ & $\begin{array}{l}\text { rapport au } \\
\text { siècle limité au } \\
\text { minimum : } \\
\text { voyages et } \\
\text { sorties à } \\
\text { l'extérieur en } \\
\text { cas de forte } \\
\text { nécessité ; rejet } \\
\text { des lä́cs à } \\
\text { l'extérieur de } \\
\text { l'enclos }\end{array}$ \\
\hline
\end{tabular}

Tableau 2 : types idéaux des chanoines (1) et des moines (2) au regard de l'urbanisation

À partir de là, les conditions matérielles de ces deux types de communautés accroissent singulièrement leurs différences du point de vue de la ville. Alors que les communautés canoniales n'hésitent pas à recourir aux services offerts par la présence d'une population laïque, les communautés bénédictines cherchent à assumer au maximum leurs besoins, privilégiant un auto-fonctionnement. Les produits exigés par une communauté canoniale sont plus nombreux, mais surtout plus variés et plus riches, que ce soit pour l'alimentation ou les vêtements : faire face à de tels besoins de consommation implique un approvisionnement plus développé 
Noizet, Hélène, "La fabrique urbaine de Tours : une analyse dialectique entre sociétés et espaces urbains (IXe-XIII" siècle)", dans V oisinages, coexistences, appropriations. Groupes sociaux et territoires urbains (Moyen Âge - 16 siècle), édité par Chloé Deligne et Claire Billen, p. 19-37, Turnhout, Brepols, 2007.

et plus complexe que pour les seuls moines, et donc des structures matérielles plus denses et diversifiées. De plus, le fait de vivre dans sa propre maison, et non pas dans un dortoir ou dans un bâtiment commun, encourage le processus d'urbanisation: le claustrum canonial comporte ainsi une organisation viaire et un parcellaire, qui n'existe pas dans l'enclos monastique. Ce dernier possède en général des dimensions plus vastes que le quartier canonial. L'enclos monastique gèle ainsi une portion non négligeable du sol urbain, cette portion, qui est en quelque sorte mise entre parenthèses, échappant au processus de l'urbanisation au moins jusqu'à la fin du XVIIIe siècle, voire jusqu'à aujourd'hui. Le rapport au siècle augmente encore ces différences puisqu'il est réduit au strict minimum chez les moines, alors que les chanoines sont au contraire en contact permanent avec les laïcs. Enfin, l'absence totale d'obligation de travail pour les chanoines les oblige à recourir d'autant plus à une main-d'œuvre laïque externe, tandis que les moines, s'ils ont également besoin de serviteurs laïcs, assurent eux-mêmes une partie de leurs besoins. La population laïque qui gravite autour d'une communauté canoniale est donc proportionnellement plus importante qu'autour d'un monastère, favorisant le processus de fabrique urbaine puisqu'il faut bien loger et nourrir tous ces gens. Ainsi, les structures sociospatiales suscitées par l'idéal-type canonial sont nécessairement plus denses et complexes que celles engendrées par l'idéal-type monastique.

Précisons que le cas de Tours permet d'épurer au maximum ces types idéaux à cause de l'imbrication des deux espaces de Saint-Martin et de Saint-Julien. Si les composantes dominantes de ces types idéaux font que les chanoines, à l'inverse des moines, peuvent être considérés comme des fabricants de la ville, dans la réalité, ces types produisent toujours des espaces mixtes: d'une part, les communautés monastiques fabriquent en partie un espace urbain, celui dans lequel se trouvent leurs serviteurs laïcs, d'autre part, les communautés canoniales fabriquent autour de leur cloître un espace peu densément structuré. Étant donné qu'à Tours, les structures de Saint-Julien potentiellement denses ont été reportées sur Châteauneuf, ces types idéaux s'opposent parfaitement.

La comparaison faite avec la ville de Saint-Denis, réalisée grâce à la documentation réunie par l'équipe des archéologues de Saint-Denis réunie autour de M. Wyss ${ }^{22}$, semble confirmer la validité de ces outils.

$\mathrm{Au}$ moment où les frères de Saint-Martin adoptaient la règle canoniale, au début du IX $\mathrm{IX}^{\mathrm{e}}$ siècle, les frères de Saint-Denis faisaient quant à eux le choix de la règle bénédictine. Or l'analyse morphologique de l'espace urbain de Saint-Denis permet effectivement de mettre en évidence que le degré d'urbanisation de cette ville est globalement assez faible, et notamment plus faible qu'à Tours. À partir des critères urbains de la diversité et de la densité parcellaires, la structure de cette ville peut être divisée en trois ensembles différents. Tout d'abord, l'enclos monastique lui-même, qui se trouve dans au sud-est de l'espace enclos par la muraille de la fin du Moyen Âge, occupe une grande surface de la ville intra muros: force est de constater que cette emprise a gelé une portion non négligeable de la ville. Cet enclos inclut toutes les infrastructures nécessaires au bon fonctionnement de la communauté monastique de Saint-Denis, à savoir, outre les édifices conventuels eux-mêmes, la

22 WYss M. (dir.), Atlas historique de Saint-Denis. Des origines au XVIII siècle, DAF, 59, Paris, 1996, figure 251, p. 281. 
Noizet, Hélène, "La fabrique urbaine de Tours : une analyse dialectique entre sociétés et espaces urbains (IXe-XIII ${ }^{\mathrm{e}}$ siècle)", dans Voisinages, coexistences, appropriations. Groupes sociaux et territoires urbains (Moyen Âge - 16e siècle), édité par Chloé Deligne et Claire Billen, p. 19-37, Turnhout, Brepols, 2007.

boulangerie, des ateliers, mais également des jardins, des vergers, sans parler des aménagements hydrographiques liés à la dérivation du Croult, dont le bras sud traverse l'enclos ${ }^{23}$. L'auto-fonctionnement monastique, recherché par la norme bénédictine, a soustrait ce secteur du processus de l'urbanisation. Au nord de l'enclos monastique, se trouve le seul espace que l'on peut véritablement qualifier d'urbain : il comporte manifestement les plus fortes densités parcellaires. Comme à Châteauneuf, le nombre des îlots y est le plus élevé de toute la ville, de même que les tailles des îlots figurent parmi les plus petites. À l'intérieur des îlots, les parcelles sont de petites dimensions et ouvrent toutes directement sur une rue. Il n'y a donc pas de grande parcelle enclavée au milieu des îlots : les fonds de parcelles ne jouxtent que d'autres fonds de parcelles, qui donnent elles-mêmes sur une autre rue. On retrouve même une esquisse de réseau viaire labyrinthique, dans le secteur des Trois-Patrons, comme c'était le cas à proximité de la place de Châteauneuf à Tours. Mais cet ensemble ne recouvre au total qu'un secteur limité par rapport à l'ensemble de l'espace intra muros. Au contraire, le troisième et dernier ensemble, qui est le moins urbain, est aussi le plus vaste: il s'étend dans les parties sud, ouest et nord de l'espace enclos au XIVe siècle. Ce parcellaire ressemble tout à fait à ce qui a été repéré autour de Saint-Julien à Tours : ici, l'urbanisation s'est limitée aux fronts de rue, comme le montre le parcellaire laniéré et oblong de part et d'autre des deux anciennes rues partant de la place du Châtelet, et allant, l'une au sud vers la place à proximité des Récollets (rue de la Boulangerie), et l'autre à l'ouest, vers Saint-Denisde-l'Estrée (rue Compoise). Mais l'urbanisation s'est arrêtée là : le tissu n'a pas été urbanisé en profondeur, puisque derrière ce rideau plus ou moins continu de petites parcelles, on trouve d'immenses parcelles, qui sont certainement d'anciens champs ou terrains vagues non colonisés par l'habitat. Pas moins de cinq communautés bénédictines se sont installées à l'époque moderne sur ces grandes parcelles : les Visitandines, les Ursulines, les Récollets, les Annonciades et les Carmélites. Leur installation, permise par l'existence de ces terrains dégagés, c'est-à-dire par la structure matérielle, a renforcé la mono-activité monastique et a également participé à réactualiser la faible densité du parcellaire : la mise en place de ces couvents a ainsi contribué à pérenniser la structure lâche de l'espace urbain.

\section{CONCLUSION}

Dans tous les cas, c'est bien l'interaction permanente de l'espace urbain et de ces multiples configurations sociales, historiquement situées, qui a abouti à un tel paysage urbain. Ce processus, appelé fabrique urbaine, est impensé, au sens où il ne résulte pas d'une démarche volontariste et consciente. S'il existe bien une articulation entre les projets des habitants et l'espace urbain, j'espère avoir montré qu'elle était dialectique, et non pas mécanique: c'est dire qu'elle ne peut être analysée au niveau de l'intentionnalité des acteurs et de l'échelle du projet humain, mais au niveau du temps long du processus, observé ici sur plus de quatre siècles. Un des éléments moteurs de cette relation dialectique semble résider dans le choix de la règle de vie, canoniale ou monastique : les deux types idéaux des chanoines et

23 Wyss M. (dir.), Atlas historique de Saint-Denis..., op. cit., pp. 138-141 et p. 321 pour le Croult. 
Noizet, Hélène, "La fabrique urbaine de Tours : une analyse dialectique entre sociétés et espaces urbains (IXe-XIII" siècle)", dans Voisinages, coexistences, appropriations. Groupes sociaux et territoires urbains (Moyen Âge - 16 siècle), édité par Chloé Deligne et Claire Billen, p. 19-37, Turnhout, Brepols, 2007.

des moines expliquent pourquoi certains quartiers sont plus urbains que d'autres. Il est d'ailleurs remarquable qu'aujourd'hui encore, les Tourangeaux partagent tous l'idée que le «Vieux Tours » correspond au quartier de Châteauneuf, et non pas à la cathédrale, ni même à Saint-Julien. La centralité urbaine n'est ni historique - ce n'est pas l'espace le plus ancien qui est le centre -, ni géographique - il n'existe aucune position idéale plus déterminante qu'une autre : tout en restant au bord de la Loire, le site de Tours aurait pu être plus en amont ou en aval, ou encore sur l'autre rive -, mais sociale: le centre urbain est bien l'espace qui a enregistré dans sa structure matérielle le plus grand nombre d'actions sociales au cours du temps.

\section{Liste des figures}

1. Plan 1 : topographie des quartiers Saint-Martin et Saint-Julien de Tours (cartographie H. Noizet, UMR 6173).

2. Plan 2: les trois secteurs urbains considérés de la ville de Tours (cartographie H. Noizet, UMR 6173).

3. Plan 3: cadastre napoléonien de l'intérieur d'îlot du secteur de Saint-Julien

4. Plan 4 : l'organisation parcellaire d'un îlot du secteur de Saint-Julien d'après le cadastre napoléonien

5. Plan 5: caractéristiques parcellaires du cloître de Saint-Martin (cadastre napoléonien)

6. Plan 6: caractéristiques parcellaires du secteur de Châteauneuf (cadastre napoléonien)

7. Tableau 1 et diagramme 1 : comparaison des profils spatiaux dans trois quartiers tourangeaux

8. Plan 7: parcellaire à l'intérieur du castrum de Saint-Martin (cadastre napoléonien).

9. Tableau 2 : types idéaux des chanoines (1) et des moines (2) au regard de l'urbanisation 


\section{Figure 1 - Topographie de la ville de Tours}

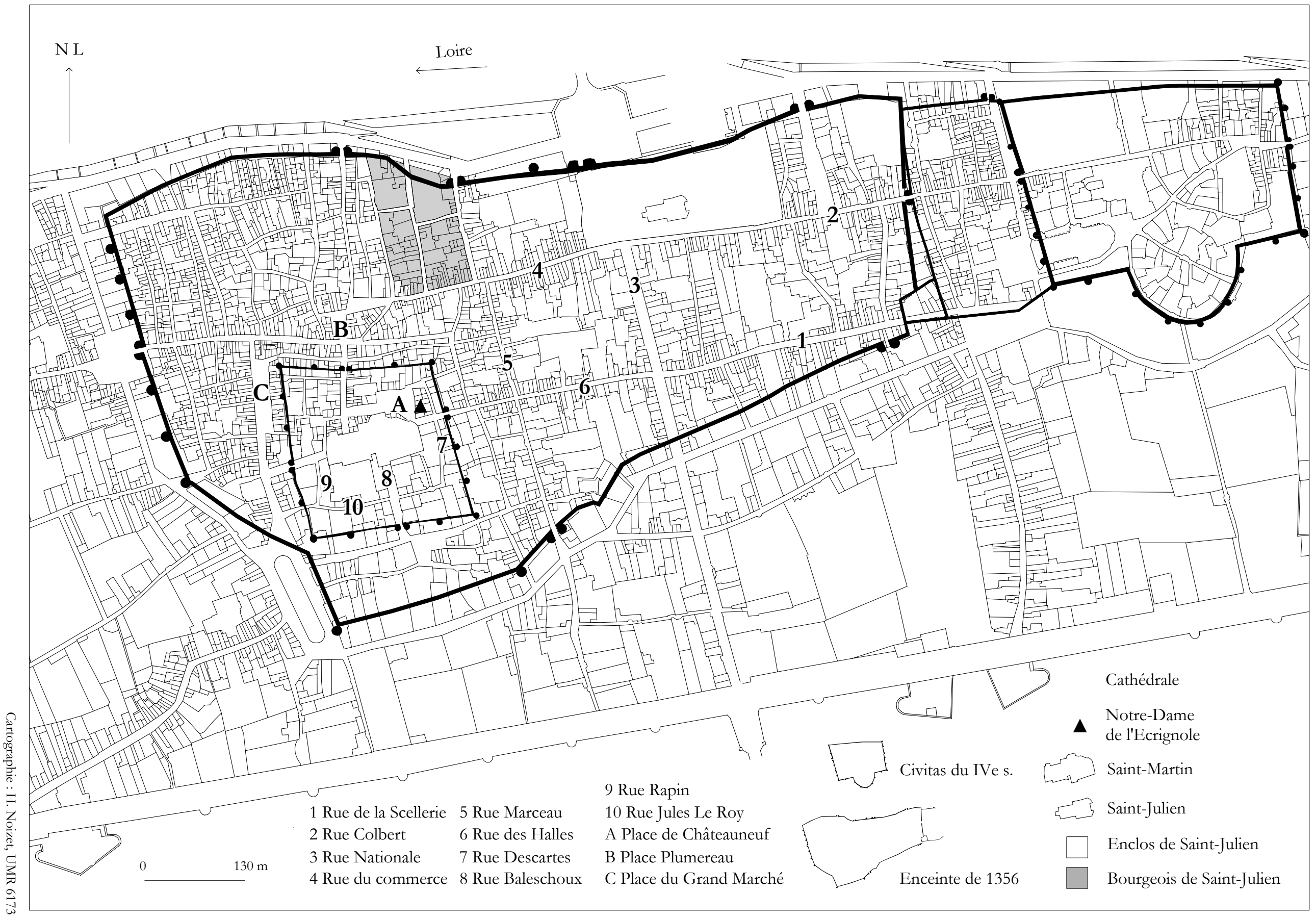

Noizet Hélène, "La fabrique urbaine de Tours : une analyse dialectique entre sociétés et espaces urbains (IXe-XIIIe siècle)", dans Voisinages, coexistences, appropriations. Groupes sociaux et territoires urbains (Moyen Âge - 16e siècle), édité par Chloé Deligne et Claire Billen, p. 19-37, Turnhout, Brepols, 2007. 
Figure 2 - Les trois secteurs urbains de la ville de Tours

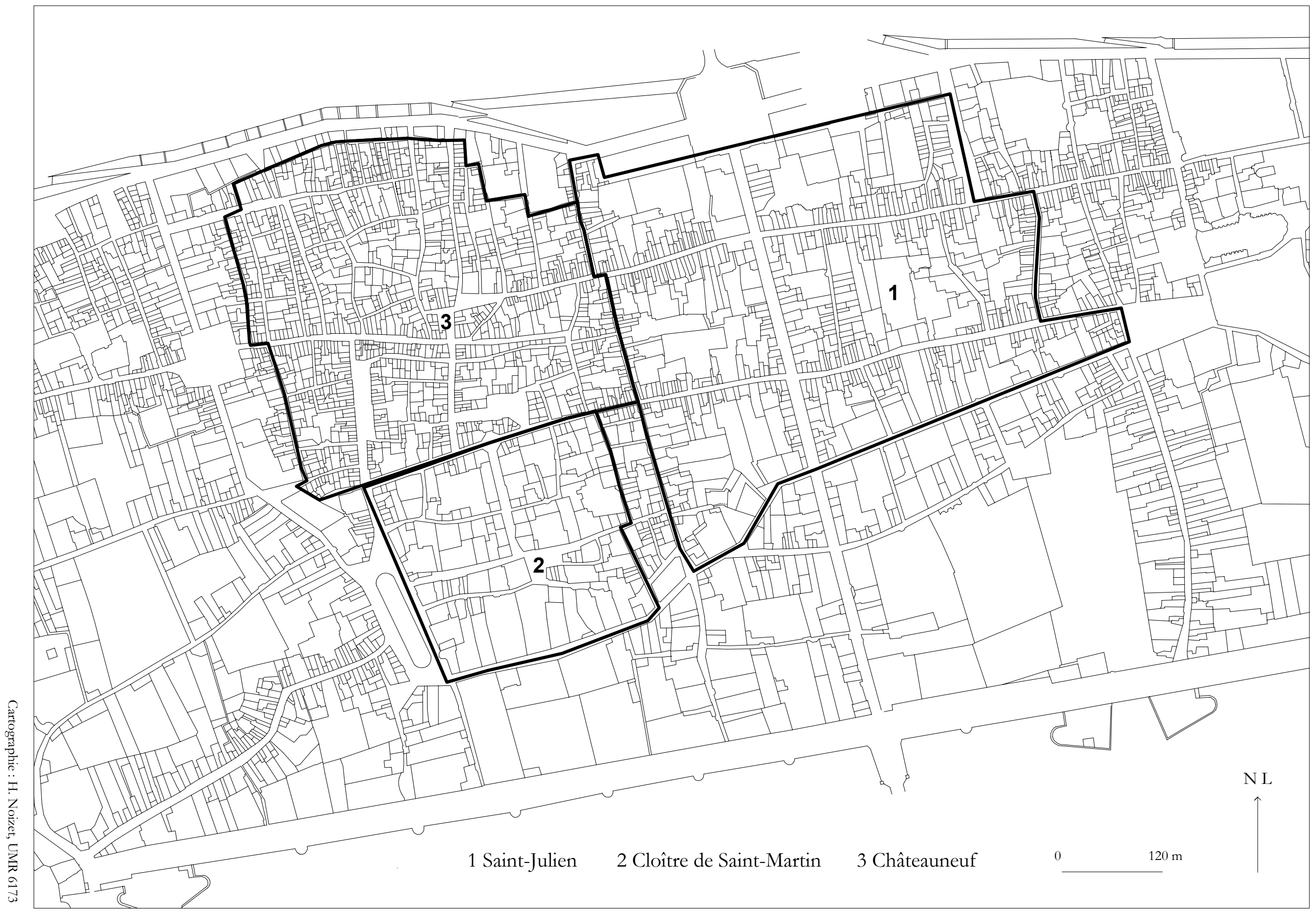

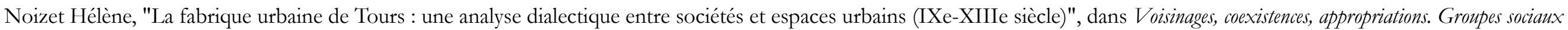
et territoires urbains (Moyen Âge - 16e siècle), édité par Chloé Deligne et Claire Billen, p. 19-37, Turnhout, Brepols, 2007. 


\section{Figure 3 - Cadastre napoléonien du secteur de Saint-Julien}

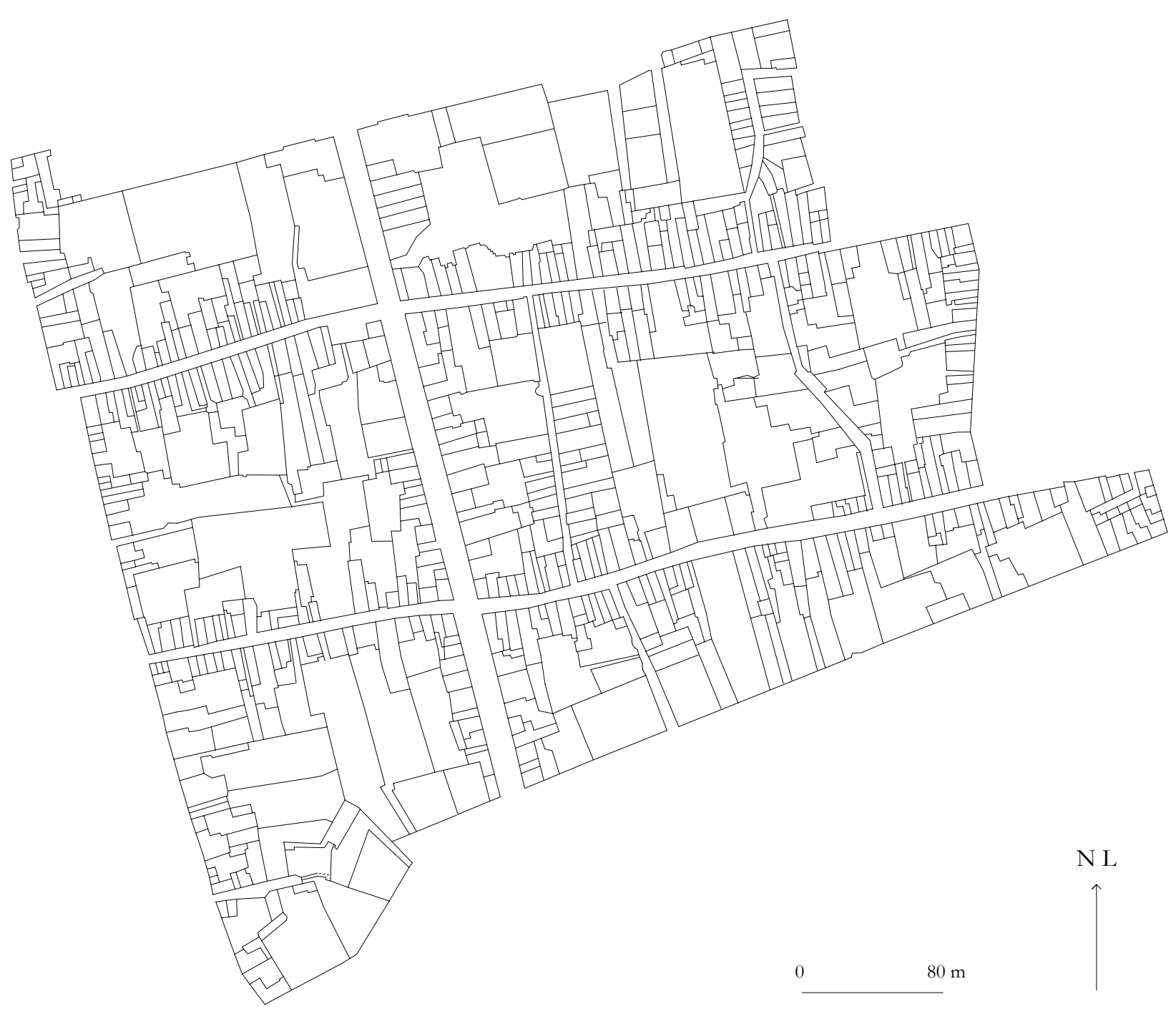

Réalisation graphique : H. Noizet, UMR 6173

Noizet Hélène, "La fabrique urbaine de Tours : une analyse dialectique entre sociétés et espaces urbains (IXe-XIIIe siècle)", dans Voisinages, coexistences, appropriations. Groupes sociaux et territoires urbains (Moyen Âge - 16e siècle), édité par Chloé Deligne et Claire Billen, p. 19-37, Turnhout, Brepols, 2007. 
Figure 4 - L'organisation parcellaire d'un îlot du secteur de Saint-Julien

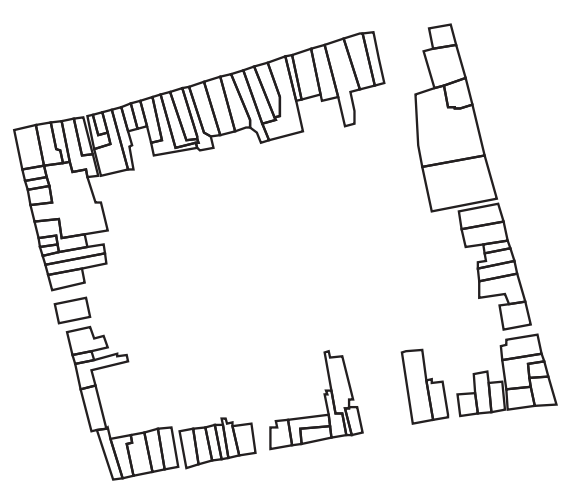

Franges de l'îlot

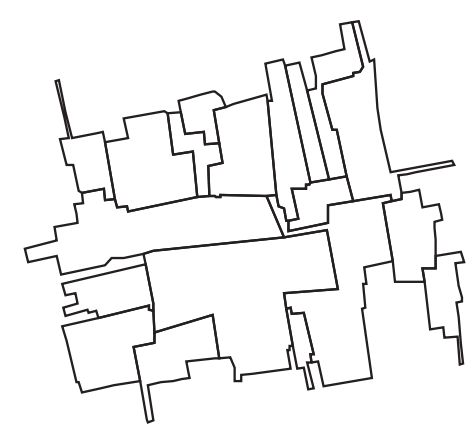

Centre de l'îlot $\stackrel{\text { N L }}{\uparrow}$

$0 \quad 75 \mathrm{~m}$

Réalisation graphique : H. Noizet, UMR 6173

Noizet Hélène, "La fabrique urbaine de Tours : une analyse dialectique entre sociétés et espaces urbains (IXe-XIIIe siècle)", dans Voisinages, coexistences, appropriations. Groupes sociaux et territoires urbains (Moyen Âge - 16e siècle), édité par Chloé Deligne et Claire Billen, p. 19-37, Turnhout, Brepols, 2007. 


\section{Figure 5 - Cadastre napoléonien du cloître de Saint-Martin}

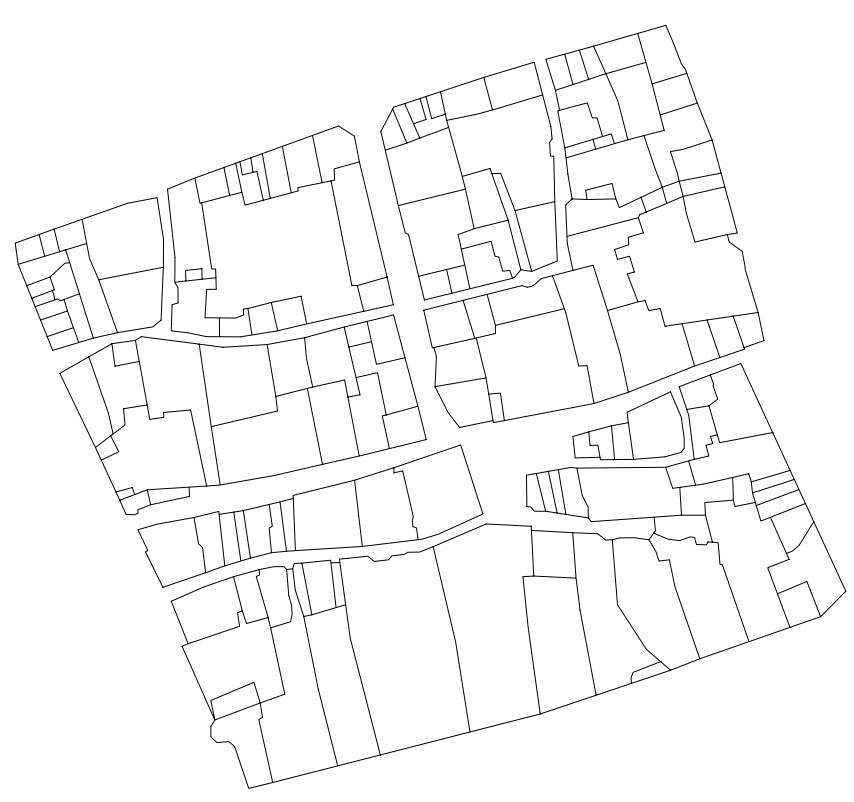

0

Noizet Hélène, "La fabrique urbaine de Tours : une analyse dialectique entre sociétés et espaces urbains (IXe-XIIIe siècle)", dans Voisinages, coexistences, appropriations. Groupes sociaux et territoires urbains (Moyen Age - 16e siècle), édité par Chloé Deligne et Claire Billen, p. 19-37, Turnhout, Brepols, 2007. 
Figure 6 - Cadastre napoléonien de Châteauneuf

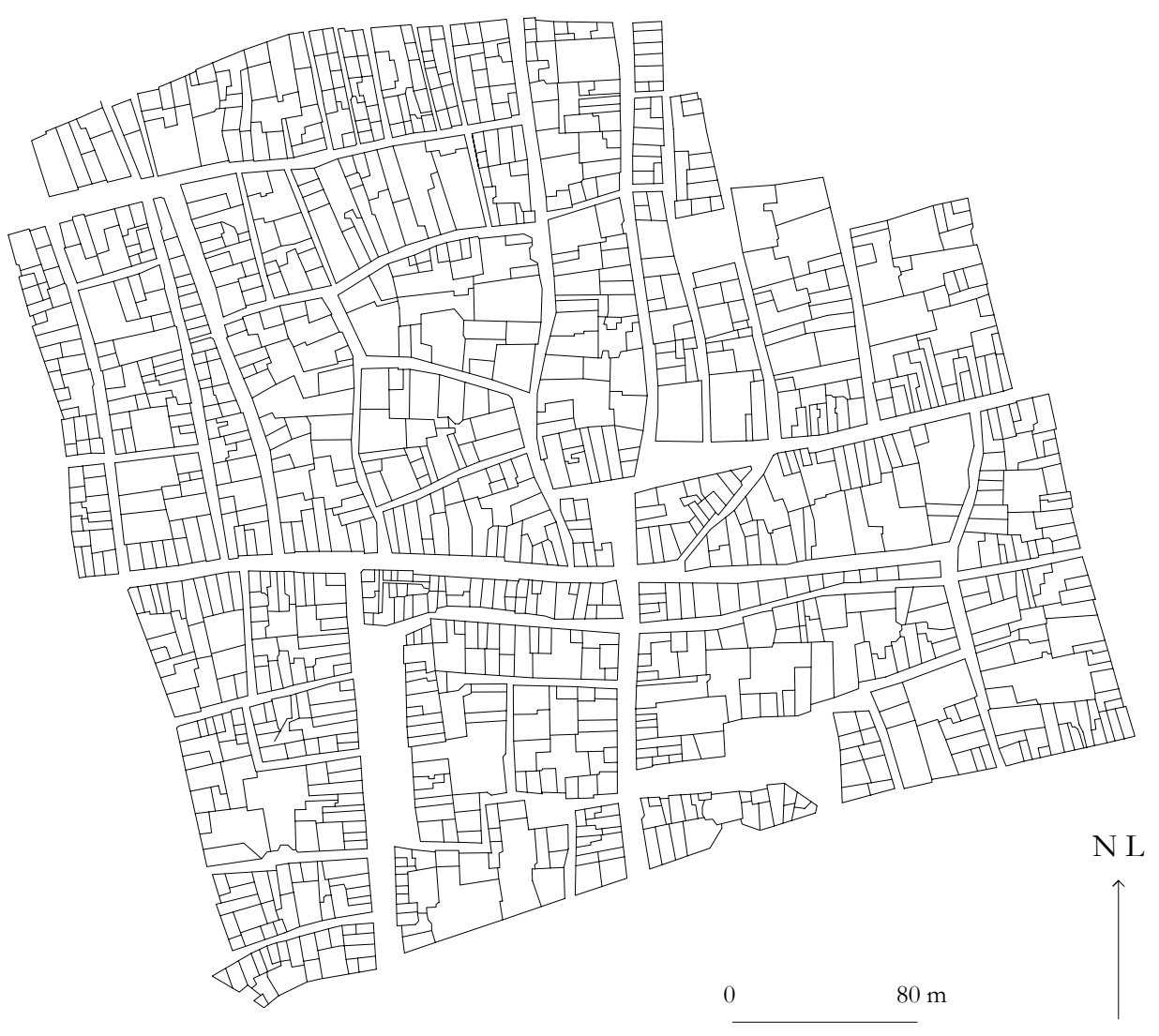

Réalisation graphique : H. Noizet, UMR 6173

Noizet Hélène, "La fabrique urbaine de Tours : une analyse dialectique entre sociétés et espaces urbains (IXe-XIIIe siècle)", dans Voisinages, coexistences, appropriations. Groupes sociaux et territoires urbains (Moyen Age - 16e siècle), édité par Chloé Deligne et Claire Billen, p. 19-37, Turnhout, Brepols, 2007. 


\section{Figure 8 - Parcellaire du castrum de Saint-Martin}
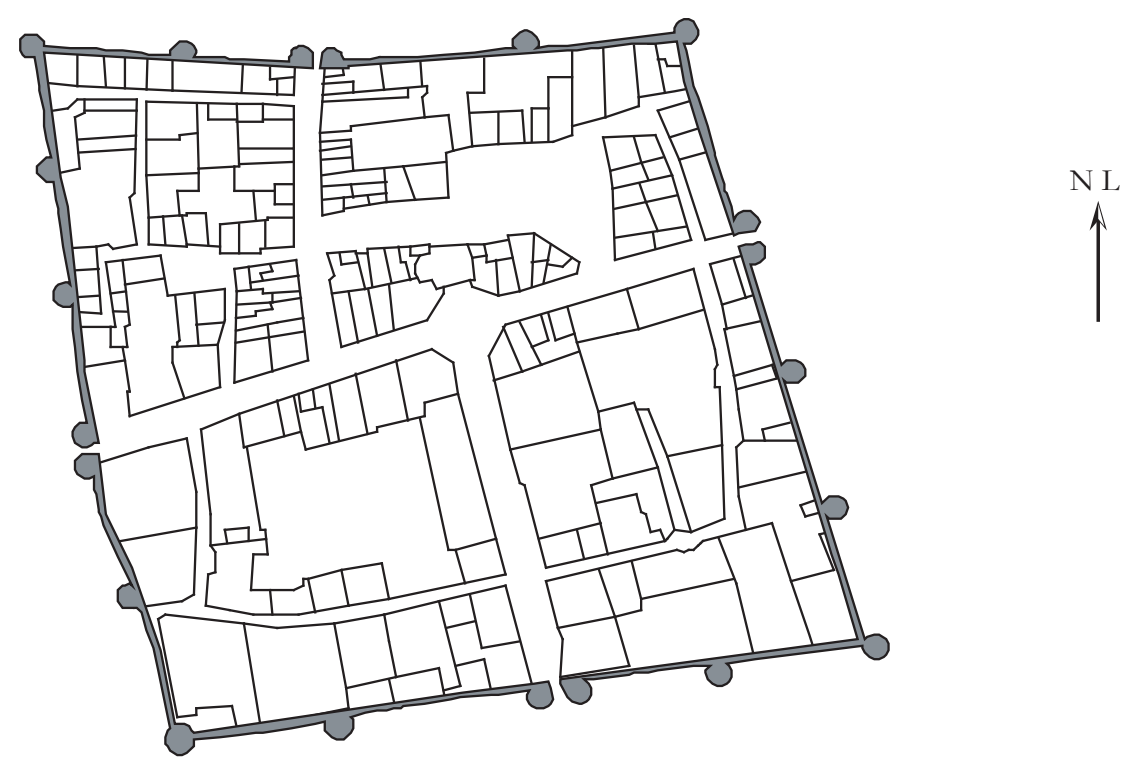

0

$75 \mathrm{~m}$

Réalisation graphique : H. Noizet, UMR 6173

Noizet Hélène, "La fabrique urbaine de Tours : une analyse dialectique entre sociétés et espaces urbains (IXe-XIIIe siècle)", dans Voisinages, coexistences, appropriations. Groupes sociaux et territoires urbains (Moyen Âge - 16e siècle), édité par Chloé Deligne et Claire Billen, p. 19-37, Turnhout, Brepols, 2007. 